\title{
The Helium Ion Microscope - a Versatile Tool for a Wide Range of Applications
}

\author{
Bernhard Goetze, Chuong Huynh, Lewis Stern, Huimeng Wu, David Ferranti and Mohan Ananth \\ Carl Zeiss Microscopy LLC, IMIC Ion Microscopy Innovation Center, Peabody, MA USA
}

Since the commercialization of the Helium Ion Microscope, the technology has been successfully used for a wide variety of applications. This talk will give an overview of areas in which Helium-imaging and the use of Neon for sample-manipulation has proven to give new insights. Advances have been made in determining the porosity of rock and shale for the oil and gas industry. The fabrication of $<5 \mathrm{mn}$ Nanopores for next generation sequencing devices using the Helium beam has proven to be more effective in comparison to electron beams. In the field of biomedical research, imaging of uncoated specimen is revealing new information about the ultra-structure of cells and tissues.

In recent years the exploitation of oil and gas reservoirs previously out of reach for conventional extraction techniques has been largely expanded. Two important parameters for the exploitability of a geological structure are the pore sizes on the nm scale as well as the connectivity of those pores. The use of a Helium beam provides high resolution images allowing for a more precise analysis of the pore sizes down to $2 \mathrm{~nm}$. Interconnected pores are pathways for possible gas transport and can be identified due to the large depth of field of the Helium Ion Microscope. No special sample preparation is necessary to investigate these samples, making their turnover extremely fast and reliable. [1]

Editing circuits on computer chips is an application with highest demands on precision in manipulating structures as well as the ability to fabricate structures on the $\mathrm{nm}$ scale. The use of Neon instead of the conventional Ga-beams yields more precise material removal without implantation-artifacts. The size of structures created using the Helium beam and metal precursors for circuit repair and editing are small enough to meet the high requirements of the next generation chip designs. We demonstrate the reliable fabrication of metal nano-wires with a width of $30 \mathrm{~nm}$ and a $20 \mathrm{~nm}$ pitch. Figure 1, [3].

The three dimensional ultrastructure of a biological sample is often linked to its function and therefore investigation of those structures gives insights in basic functionality of cells and tissues. Due to the charge compensation mechanism of the Helium Ion Microscope, biological specimen can now be investigated without applying a conductive coating while making full use of the resolving power of the instrument. [Figure 3] The combination of gentle sample preparation protocols and Helium Ion Microscopy has shown remarkable preservation of surface features. We present insights in the 3 dimensional ultrastructure of the renal filtration barrier of the glomerolus and surface features of cells in the collecting duct.[2]

The investigation of a defined area within a tissue or cell has been limited in the past to Microtomesections which can be oriented sub optimally. We demonstrate the use of a focused ion beam in order to place precisely directed cuts into biological tissue. The distal part of the pharynx of a ground worm (Pristionchus pacificus) was removed using a Neon beam in order to investigate the structures of the feeding apparatus. [Figure 3]

The wide variety of applications that can be covered with a Helium Ion Microscope makes it an ideal tool for imaging centers serving users from several disciplines. 


\section{References:}

[1] C Kliewer et al, GIT Imaging \& Microscopy volume 3 (2012) p. 15.

[2] Rice et al, PLOS ONE in press

[3] Stern and $\mathrm{Wu}$, unpublished

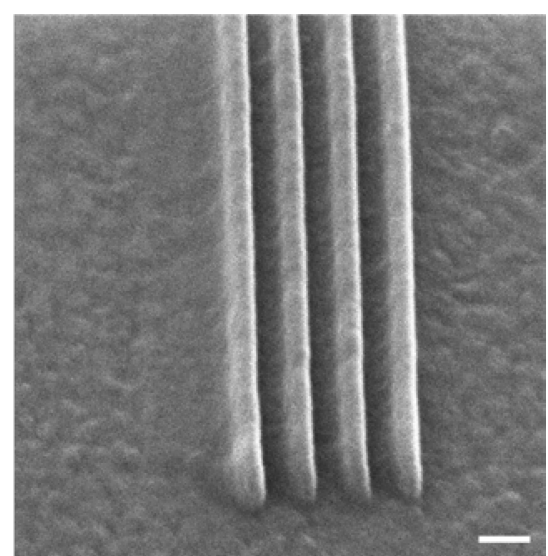

Figure 1

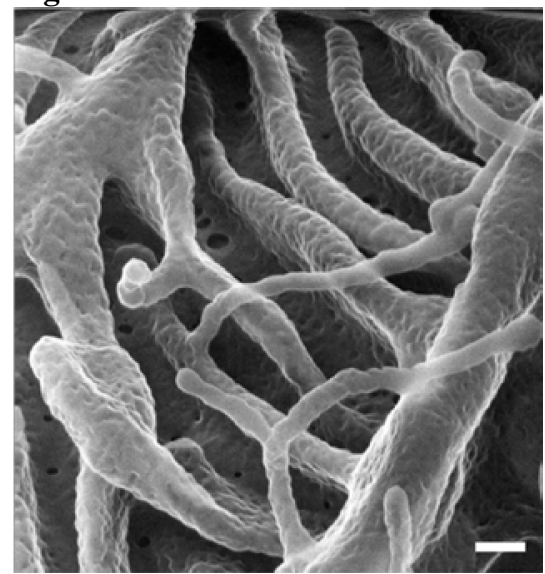

Figure 3A

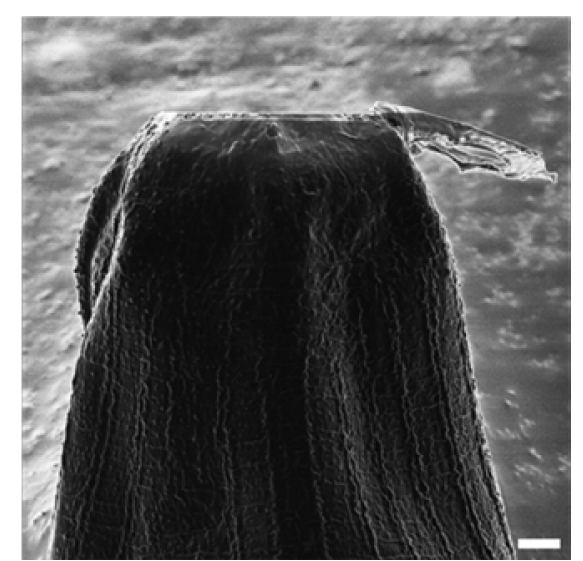

Figure 2

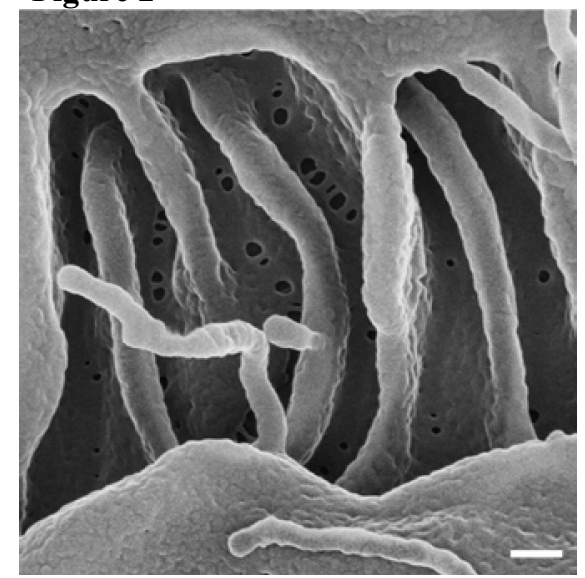

Figure 3B

Figure 1. Metal nanowires, $30 \mathrm{~nm}$ in width and with a $20 \mathrm{~nm}$ pitch, deposited using a Helium beam. Scale bar: $50 \mathrm{~nm}$.

Figure 2. Ground worm (Pristionchus Pacificus) cut open using a Neon beam in order to expose the distal part of the pharynx. The image has been taken with an Orion Ion Microscope using Neon as a gas source. Sample preparation and imaging has been performed in collaboration with J. Fitzpatrick and Matthew Joens, Waitt Advanced Biophotonics Center of the Salk Institute, La Jolla, CA. Scale bar $2 \mu \mathrm{m}$

Figure 3. Podocytes in the rat kidney imaged with Helium Ion Microscopy. The pores of the slit diaphragm are clearly visible. Protrusions from the surface of the podocytes are preserved. (A) Uncoated sample. (B) A $3 \mathrm{~nm} \mathrm{Pt} / \mathrm{Pd}$ metal coating has been applied. The native surface of the podocytes is obscured by the coating and only the grains of the coating are visible. Also the pore sizes of the slit diaphragm are reduced due to the metal deposition.

Scale bars $100 \mathrm{~nm}$ 